\title{
Exploring the evolution of color-luminosity parameter $\beta$ and its effects on parameter estimation
}

\author{
Shuang Wang, ${ }^{1, *}$ * Yun-He Li,, , \\ ${ }^{1}$ Department of Physics, College of Sciences, Northeastern University, Shenyang 110004, China \\ ${ }^{2}$ Center for High Energy Physics, Peking University, Beijing 100080, China
}

\begin{abstract}
It has been found in previous studies that, for the Supernova Legacy Survey three-year (SNLS3) data, there is strong evidence for the redshift-evolution of color-luminosity parameter $\beta$. In this paper, using three simplest dark energy models ( $\Lambda$ CDM, $w \mathrm{CDM}$, and CPL), we further explore the evolution of $\beta$ and its effects on parameter estimation. In addition to the SNLS3 data, we also take into account the Planck distance priors data, as well as the latest galaxy clustering (GC) data extracted from SDSS DR7 and BOSS. We find that, for all the models, adding a parameter of $\beta$ can reduce $\chi_{\min }^{2}$ by $\sim 36$, indicating that $\beta_{1}=0$ is ruled out at $6 \sigma$ confidence levels. In other words, $\beta$ deviates from a constant at $6 \sigma$ confidence levels. This conclusion is insensitive to the dark energy models considered, showing the importance of considering the evolution of $\beta$ in the cosmology-fits. Furthermore, it is found that varying $\beta$ can significantly change the fitting results of various cosmological parameters: using the SNLS3 data alone, varying $\beta$ yields a larger $\Omega_{m}$ for the $\Lambda$ CDM model; using the SNLS3+CMB+GC data, varying $\beta$ yields a larger $\Omega_{m}$ and a smaller $h$ for all the models. Moreover, we find that these results are much closer to those given by the $\mathrm{CMB}+\mathrm{GC}$ data, compared to the cases of treating $\beta$ as a constant. This indicates that considering the evolution of $\beta$ is very helpful for reducing the tension between supernova and other cosmological observations.
\end{abstract}

PACS numbers: 98.80.-k, 98.80.Es, 95.36.+x

Keywords: Cosmology, type Ia supernova, dark energy

\section{INTRODUCTION}

Various astronomical observations [1/7] all indicate that the Universe is undergoing an accelerated expansion. So far, we are still in the dark about the nature of this extremely counterintuitive phenomenon; it may be due to an unknown energy component, i.e., dark energy 8 19], or a modification of general relativity [20 27]. For recent reviews, see, e.g., 28 37.

One of the most powerful probes of dark energy is the use of type Ia supernovae (SNe Ia), which can be used as cosmological standard candles to measure the expansion history of the Universe. In recent years, several supernova $(\mathrm{SN})$ datasets with hundreds of SNe Ia were released, such as "SNLS" 38, "Union" 39, "Constitution" 40], "SDSS" 41], "Union2" 42] and "Union2.1" 43].

In 2010, a high quality SN dataset from the first three years of the Supernova Legacy Survey (SNLS3) was released 44. Soon after, Conley et al. presented SNe-only cosmological results by combining the SNLS3 SNe with various low- to mid- $z$ samples [45, and Sullivan et al. presented the joint cosmological constraints by combining the SNLS3 dataset with other cosmological data sets [46]. In 45] three $\mathrm{SNe}$ data sets are presented, depending on different light-curve fitters: "SALT2", which con-

\footnotetext{
${ }^{\ddagger}$ Corresponding author

*Electronic address: wjysysgj@163.com

${ }^{\dagger}$ Electronic address: liyh19881206@126.com

$\S$ Electronic address: zhangxin@mail.neu.edu.cn
}

sists of 473 SNe Ia; "SiFTO", which consists of 468 SNe Ia; and "combined", which consists of 472 SNe Ia. It should be stressed that the SNLS team treated two important quantities, stretch-luminosity parameter $\alpha$ and color-luminosity parameter $\beta$ of SNe Ia, as free model parameters on the same footing as the cosmological parameters, all to be estimated during the Hubble diagram fitting process using the covariance matrix that includes both statistical and systematic errors.

A critical challenge is the control of the systematic uncertainties of SNe Ia. One of the most important factors is the effect of potential SN evolution, i.e., the possibility of the evolution of $\alpha$ and $\beta$ with redshift $z$. The current studies show that $\alpha$ is still consistent with a constant, but the hints for the evolution of $\beta$ have already been found in [38, 39, 41, 44, 47]. It should be pointed out that these papers studied $\beta$ 's evolution using binby-bin fits, which were very difficult to make definitive statements because of the correlations between different bins. In 48, Mohlabeng and Ralston firstly used a linear parametrization $\beta(z)=\beta_{0}+\beta_{1} z$ to study the Union2.1 sample, and found that $\beta$ deviates from a constant at $7 \sigma$ confidence levels. Moreover, they proved that using the linear parametrization can obtain better results than using bin-by-bin methods. In [49], Wang and Wang studied the case of SNLS3 data using three functional forms: the linear, the quadratic, and the step function fits. They found that $\beta$ increases significantly with $z$ when the systematic uncertainties of SNLS3 sample are taken into account, showing that the evolution of $\beta$ is insensitive to the functional form of $\beta(z)$ assumed.

It is clear that a time-varying $\beta$ has significant impact 
on parameter estimation. In [49, using the cubic spline interpolation for a scaled comoving distance $r_{p}(z)$, the effects of varying $\beta$ on distance-redshift relation is briefly discussed. It is also very interesting to study the impact of varying $\beta$ on various cosmological models. So in this paper, we study this issue by considering three simplest dark energy models: $\Lambda \mathrm{CDM}, w \mathrm{CDM}$, and CPL [50]. For comparison, we also take into account the Planck distance priors data 51, as well as the latest galaxy clustering (GC) data extracted from SDSS DR7 [52] and BOSS [53].

We describe our method in Sec. II, present our results in Sec. III, and conclude in Sec. IV.

\section{METHOD}

The comoving distance to an object at redshift $z$ is given by:

$$
\begin{aligned}
& r(z)=c H_{0}^{-1}\left|\Omega_{k}\right|^{-1 / 2} \operatorname{sinn}\left[\left|\Omega_{k}\right|^{1 / 2} \Gamma(z)\right], \\
& \Gamma(z)=\int_{0}^{z} \frac{d z^{\prime}}{E\left(z^{\prime}\right)}, \quad E(z)=H(z) / H_{0}
\end{aligned}
$$

where $\operatorname{sinn}(x)=\sin (x), x, \sinh (x)$ for $\Omega_{k}<0, \Omega_{k}=$ 0 , and $\Omega_{k}>0$ respectively. The expansion rate of the universe $H(z)$ (i.e., the Hubble parameter) is given by

$$
H^{2}(z)=H_{0}^{2}\left[\Omega_{m}(1+z)^{3}+\Omega_{k}(1+z)^{2}+\Omega_{X} X(z)\right],
$$

where $\Omega_{m}+\Omega_{k}+\Omega_{X}=1 . \Omega_{m}$ also includes the contribution from massive neutrinos besides the contributions from baryons and dark matter; the dark energy density function $X(z)$ is defined as

$$
X(z) \equiv \frac{\rho_{X}(z)}{\rho_{X}(0)} .
$$

Note that $\Omega_{\text {rad }}=\Omega_{m} /\left(1+z_{\text {eq }}\right) \ll \Omega_{m}$ (with $z_{\text {eq }}$ denoting the redshift at matter-radiation equality), thus the $\Omega_{\mathrm{rad}}$ term is usually omitted in dark energy studies at $z \ll$ 1000 , since dark energy should only be important at the late times.

\section{A. SNe Ia data}

SNe Ia data give measurements of the luminosity distance $d_{L}(z)$ through that of the distance modulus of each $\mathrm{SN}$ :

$$
\mu_{0} \equiv m-M=5 \log \left[\frac{d_{L}(z)}{\mathrm{Mpc}}\right]+25,
$$

where $m$ and $M$ represent the apparent and absolute magnitude of an SN. The luminosity distance $d_{L}(z)=$ $(1+z) r(z)$, with the comoving distance $r(z)$ given by Eq. (1).

Here we use the SNLS3 data set. As mentioned above, based on different light-curve fitters, three $\mathrm{SNe}$ sets of
SNLS3 are given, including "SALT2", "SiFTO", and "combined". As shown in [49, the conclusion of evolution of $\beta$ is insensitive to the lightcurve fitter used to derive the SNLS3 sample. So in this paper we just use the "combined" set.

In [49, by considering three functional forms (linear case, quadratic case, and step function case), the possible evolution of $\alpha$ and $\beta$ is explored. It is found that, for the SNLS3 data, $\alpha$ is still consistent with a constant, but $\beta$ increases significantly with $z$. It should be stressed that this conclusion is insensitive to the functional form of $\alpha$ and $\beta$ assumed [49. So in this paper, we just adopt a constant $\alpha$ and a linear $\beta(z)=\beta_{0}+\beta_{1} z$. Now, the predicted magnitude of an $\mathrm{SN}$ becomes

$$
m_{\bmod }=5 \log _{10} \mathcal{D}_{L}(z \mid \mathbf{p})-\alpha(s-1)+\beta(z) \mathcal{C}+\mathcal{M},
$$

where $\mathcal{D}_{L}(z \mid \mathbf{p})$ is the luminosity distance multiplied by $H_{0}$ for a given set of cosmological parameters $\{\mathbf{p}\}, s$ is the stretch measure of the SN light curve shape, and $\mathcal{C}$ is the color measure for the SN. $\mathcal{M}$ is a nuisance parameter representing some combination of the absolute magnitude of a fiducial SN, $M$, and the Hubble constant, $H_{0}$. In order to include host-galaxy information in the cosmological fits, the SNLS3 sample is splitted into two samples based on host-galaxy stellar mass at $10^{10} M_{\odot}$, and $\mathcal{M}$ is allowed to be different for the two samples [45. Therefore, for the SNLS3 sample, there are two values of $\mathcal{M}$, i.e., $\mathcal{M}_{1}$ and $\mathcal{M}_{2}$ (for more details, see the subsections 3.2 and 5.8 of [45]). The method of analytically marginalizing over $\mathcal{M}$ in this case is detailedly described in Appendix $\mathrm{C}$ of [45, and the corresponding code is public (now it is a part of CosmoMC). In this work, we follow the recipe of 45$]$.

Since the time dilation part of the observed luminosity distance depends on the total redshift $z_{\text {hel }}$ (special relativistic plus cosmological), we have

$$
\mathcal{D}_{L}(z \mid \mathbf{s})=c^{-1} H_{0}\left(1+z_{\text {hel }}\right) r(z \mid \mathbf{s}),
$$

where $z$ and $z_{\text {hel }}$ are the CMB restframe and heliocentric redshifts of the SN.

For a set of $N$ SNe with correlated errors, we have [45]

$$
\chi^{2}=\Delta \mathbf{m}^{T} \cdot \mathbf{C}^{-1} \cdot \Delta \mathbf{m}
$$

where $\Delta m \equiv m_{B}-m_{\text {mod }}$ is a vector with $N$ components, $m_{B}$ is the rest-frame peak B-band magnitude of the $\mathrm{SN}$, and $\mathbf{C}$ is the $N \times N$ covariance matrix of the SNe.

The total covariance matrix is [45]

$$
\mathbf{C}=\mathbf{D}_{\text {stat }}+\mathbf{C}_{\text {stat }}+\mathbf{C}_{\text {sys }},
$$

with the diagonal part of the statistical uncertainty given by 45 .

$$
\begin{aligned}
\mathbf{D}_{\text {stat }, i i}= & \sigma_{m_{B}, i}^{2}+\sigma_{\text {int }}^{2}+\sigma_{\text {lensing }}^{2}+\sigma_{\text {host correction }}^{2} \\
& +\left[\frac{5\left(1+z_{i}\right)}{z_{i}\left(1+z_{i} / 2\right) \ln 10}\right]^{2} \sigma_{z, i}^{2} \\
& +\alpha^{2} \sigma_{s, i}^{2}+\beta\left(z_{i}\right)^{2} \sigma_{\mathcal{C}, i}^{2} \\
& +2 \alpha C_{m_{B} s, i}-2 \beta\left(z_{i}\right) C_{m_{B} \mathcal{C}, i} \\
& -2 \alpha \beta\left(z_{i}\right) C_{s \mathcal{C}, i}
\end{aligned}
$$


where $C_{m_{B} s, i}, C_{m_{B} \mathcal{C}, i}$, and $C_{s \mathcal{C}, i}$ are the covariances between $m_{B}, s$, and $\mathcal{C}$ for the $i$-th SN, $\beta_{i}=\beta\left(z_{i}\right)$ is the value of $\beta$ for the $i$-th SN. Note also that $\sigma_{z, i}^{2}$ includes a peculiar velocity residual of 0.0005 (i.e., $150 \mathrm{~km} / \mathrm{s}$ ) added in quadrature [45. In this paper we just use the values of intrinsic scatter $\sigma_{\text {int }}$ given in Table 4 of 45 . These values are obtained by making $\chi^{2} / d o f=1$. Varying $\sigma_{\text {int }}$ could have a significant impact on parameter estimation, see [54] for details.

We define $\mathbf{V} \equiv \mathbf{C}_{\text {stat }}+\mathbf{C}_{\text {sys }}$, where $\mathbf{C}_{\text {stat }}$ and $\mathbf{C}_{\text {sys }}$ are the statistical and systematic covariance matrices, respectively. After treating $\beta$ as a function of $z, \mathbf{V}$ is given in the form:

$$
\begin{aligned}
\mathbf{V}_{i j}= & V_{0, i j}+\alpha^{2} V_{a, i j}+\beta_{i} \beta_{j} V_{b, i j} \\
& +\alpha V_{0 a, i j}+\alpha V_{0 a, j i} \\
& -\beta_{j} V_{0 b, i j}-\beta_{i} V_{0 b, j i} \\
& -\alpha \beta_{j} V_{a b, i j}-\alpha \beta_{i} V_{a b, j i} .
\end{aligned}
$$

Here, $\beta_{j}=\beta\left(z_{j}\right)$ is the value of $\beta$ for the $j$-th $\mathrm{SN}$; the obvious difference from $\beta_{0}$ and $\beta_{1}$ in the form $\beta(z)=$ $\beta_{0}+\beta_{1} z$ should be noticed. It must be stressed that, while $V_{0}, V_{a}, V_{b}$, and $V_{0 a}$ are the same as the "normal" covariance matrices given by the SNLS3 data archive, $V_{0 b}$ and $V_{a b}$ are not the same as the ones given there. This is because the original matrices of SNLS3 are produced by assuming that $\beta$ is a constant. We have used the $V_{0 b}$ and $V_{a b}$ matrices for the "combined" set that are applicable when varying $\beta(z)$ (A. Conley, private communication, 2013).

In [49], it is found that the flux-averaging of SNe [5558. may be helpful to reduce the effect of varying $\beta$. It should be mentioned that the results of flux-averaging depend on the choices of redshift cut-off $z_{\text {cut }}: \beta$ still increases with $z$ when all the $\mathrm{SNe}$ are flux-averaged, and $\beta$ is consistent with being a constant when only SNe at $z \geq 0.04$ are flux-averaged [49]. Since the unknown systematic biases originate mostly from low $z \mathrm{SNe}$, fluxaveraging all SNe should lead to the least biased results. Therefore, after applying the flux-averaging method, the effect of varying $\beta$ is not completely removed. For simplicity, we do not use the flux-averaging method in this paper, and we will discuss the issue of flux-averaging in future work.

\section{B. CMB and GC data}

For CMB data, we use the latest distance priors data extracted from Planck first data release [51].

CMB give us the comoving distance to the photondecoupling surface $r\left(z_{*}\right)$, and the comoving sound horizon at photon-decoupling epoch $r_{s}\left(z_{*}\right)$. Wang and Mukherjee [59] showed that the CMB shift parameters

$$
\begin{aligned}
l_{a} & \equiv \pi r\left(z_{*}\right) / r_{s}\left(z_{*}\right), \\
R & \equiv \sqrt{\Omega_{m} H_{0}^{2}} r\left(z_{*}\right) / c
\end{aligned}
$$

together with $\omega_{b} \equiv \Omega_{b} h^{2}$, provide an efficient summary of CMB data as far as dark energy constraints go. Replacing $\omega_{b}$ with $z_{*}$ gives identical constraints when the CMB distance priors are combined with other data 60]. Using $\omega_{b}$, instead of $z_{*}$, is more appropriate in a Markov Chain Monte Carlo (MCMC) analysis in which $\omega_{b}$ is a base parameter.

The comoving sound horizon at redshift $z$ is given by

$$
\begin{aligned}
r_{s}(z) & =\int_{0}^{t} \frac{c_{s} d t^{\prime}}{a}=c H_{0}^{-1} \int_{z}^{\infty} d z^{\prime} \frac{c_{s}}{E\left(z^{\prime}\right)}, \\
& =c H_{0}^{-1} \int_{0}^{a} \frac{d a^{\prime}}{\sqrt{3\left(1+\overline{R_{b}} a^{\prime}\right) a^{\prime 4} E^{2}\left(z^{\prime}\right)}}
\end{aligned}
$$

where $a$ is the cosmic scale factor, $a=1 /(1+z)$, and $a^{4} E^{2}(z)=\Omega_{m}\left(a+a_{\mathrm{eq}}\right)+\Omega_{k} a^{2}+\Omega_{X} X(z) a^{4}$, with $a_{\mathrm{eq}}=\Omega_{\mathrm{rad}} / \Omega_{m}=1 /\left(1+z_{\mathrm{eq}}\right)$, and $z_{\mathrm{eq}}=$ $2.5 \times 10^{4} \Omega_{m} h^{2}\left(T_{\mathrm{cmb}} / 2.7 \mathrm{~K}\right)^{-4}$. The sound speed is $c_{s}=1 / \sqrt{3\left(1+\overline{R_{b}} a\right)}$, with $\overline{R_{b}} a=3 \rho_{b} /\left(4 \rho_{\gamma}\right), \overline{R_{b}}=$ $31500 \Omega_{b} h^{2}\left(T_{\mathrm{cmb}} / 2.7 \mathrm{~K}\right)^{-4}$. We take $T_{\mathrm{cmb}}=2.7255 \mathrm{~K}$.

The redshift to the photon-decoupling surface, $z_{*}$, is given by the fitting formula [61]:

$z_{*}=1048\left[1+0.00124\left(\Omega_{b} h^{2}\right)^{-0.738}\right]\left[1+g_{1}\left(\Omega_{m} h^{2}\right)^{g_{2}}\right]$,

where

$$
\begin{aligned}
& g_{1}=\frac{0.0783\left(\Omega_{b} h^{2}\right)^{-0.238}}{1+39.5\left(\Omega_{b} h^{2}\right)^{0.763}}, \\
& g_{2}=\frac{0.560}{1+21.1\left(\Omega_{b} h^{2}\right)^{1.81}} .
\end{aligned}
$$

The redshift of the drag epoch $z_{d}$ is well approximated by 62

$$
z_{d}=\frac{1291\left(\Omega_{m} h^{2}\right)^{0.251}}{1+0.659\left(\Omega_{m} h^{2}\right)^{0.828}}\left[1+b_{1}\left(\Omega_{b} h^{2}\right)^{b 2}\right],
$$

where

$$
\begin{aligned}
& b_{1}=0.313\left(\Omega_{m} h^{2}\right)^{-0.419}\left[1+0.607\left(\Omega_{m} h^{2}\right)^{0.674}\right] \\
& b_{2}=0.238\left(\Omega_{m} h^{2}\right)^{0.223} .
\end{aligned}
$$

Using the Planck+lensing +WP data, the mean values and $1 \sigma$ errors of $\left\{l_{a}, R, \omega_{b}\right\}$ are obtained [51,

$$
\begin{aligned}
& \left\langle l_{a}\right\rangle=301.57, \sigma\left(l_{a}\right)=0.18 \\
& \langle R\rangle=1.7407, \sigma(R)=0.0094 \\
& \left\langle\omega_{b}\right\rangle=0.02228, \sigma\left(\omega_{b}\right)=0.00030 .
\end{aligned}
$$

Defining $p_{1}=l_{a}\left(z_{*}\right), p_{2}=R\left(z_{*}\right)$, and $p_{3}=\omega_{b}$, the normalized covariance matrix $\operatorname{NormCov}_{C M B}\left(p_{i}, p_{j}\right)$ can be written as 51 .

$$
\left(\begin{array}{ccc}
1.0000 & 0.5250 & -0.4235 \\
0.5250 & 1.0000 & -0.6925 \\
-0.4235 & -0.6925 & 1.0000
\end{array}\right) .
$$


Then, the covariance matrix for $\left(l_{a}, R, \omega_{b}\right)$ is given by

$$
\operatorname{Cov}_{C M B}\left(p_{i}, p_{j}\right)=\sigma\left(p_{i}\right) \sigma\left(p_{j}\right) \operatorname{NormCov}_{C M B}\left(p_{i}, p_{j}\right),
$$

where $i, j=1,2,3$. The CMB data are included in our analysis by adding the following term to the $\chi^{2}$ function:

$$
\chi_{C M B}^{2}=\Delta p_{i}\left[\operatorname{Cov}_{C M B}^{-1}\left(p_{i}, p_{j}\right)\right] \Delta p_{j}, \Delta p_{i}=p_{i}-p_{i}^{\text {data }},
$$

where $p_{i}^{d a t a}$ are the mean values from Eq. 119.

For GC data, we use the measurements of $\overline{H(z)} r_{s}\left(z_{d}\right) / c$ and $D_{A}(z) / r_{s}\left(z_{d}\right)$ from the two-dimensional two-point correlation function measured at $z=0.35$ [52] and $z=$ 0.57 [53, where the angular diameter distance $D_{A}(z)=$ $c H_{0}^{-1} r(z) /(1+z)$. The $z=0.35$ measurement was made by Chuang and Wang 52] using a sample of the SDSS DR7 Luminous Red Galaxies (LRGs). The $z=0.57$ measurement was made by Chuang et al. 53. using the CMASS galaxy sample from BOSS.

Using the two-dimensional two-point correlation function of SDSS DR7 in the scale range of $40-120 \mathrm{Mpc} / h$, Chuang and Wang [52] found that

$$
\begin{aligned}
& H(z=0.35) r_{s}\left(z_{d}\right) / c=0.0434 \pm 0.0018 \\
& D_{A}(z=0.35) / r_{s}\left(z_{d}\right)=6.60 \pm 0.26
\end{aligned}
$$

where $r_{s}\left(z_{d}\right)$ is the sound horizon at the drag epoch given by Eqs. (12) and (16). In a similar analysis using the CMASS galaxy sample from BOSS, Chuang et al. 53 found that

$$
\begin{aligned}
& H(z=0.57) r_{s}\left(z_{d}\right) / c=0.0454 \pm 0.0031 \\
& D_{A}(z=0.57) / r_{s}\left(z_{d}\right)=8.95 \pm 0.27
\end{aligned}
$$

GC data are included in our analysis by adding $\chi_{G C}^{2}=$ $\chi_{G C 1}^{2}+\chi_{G C 2}^{2}$, with $z_{G C 1}=0.35$ and $z_{G C 2}=0.57$, to $\chi^{2}$ of a given model. Note that

$$
\chi_{G C i}^{2}=\Delta q_{i}\left[\mathrm{C}_{G C i}^{-1}\left(q_{i}, q_{j}\right)\right] \Delta q_{j}, \Delta q_{i}=q_{i}-q_{i}^{\text {data }},
$$

where $q_{1}=H\left(z_{G C i}\right) r_{s}\left(z_{d}\right) / c, q_{2}=D_{A}\left(z_{G C i}\right) / r_{s}\left(z_{d}\right)$, and $i=1,2$. Based on Refs. [52] and [53, we have

$$
\begin{aligned}
& \mathrm{C}_{G C 1}=\left(\begin{array}{cc}
0.00000324 & 0.0000282672 \\
0.0000282672 & 0.0676
\end{array}\right), \\
& \mathrm{C}_{G C 2}=\left(\begin{array}{cc}
0.00000961 & 0.0004079538 \\
0.0004079538 & 0.0729
\end{array}\right) .
\end{aligned}
$$

\section{RESULTS}

As mentioned above, in this paper we consider three simplest models: $\Lambda$ CDM, $w$ CDM, and CPL. To explore the evolution of color-luminosity parameter $\beta$, we study the case of constant $\alpha$ and linear $\beta(z)=\beta_{0}+\beta_{1} z$; for comparison, the case of constant $\alpha$ and constant $\beta$ is also taken into account.
We perform an MCMC likelihood analysis 63. to obtain $\mathcal{O}\left(10^{6}\right)$ samples for each set of results presented in this paper. We assume flat priors for all the parameters, and allow ranges of the parameters wide enough such that further increasing the allowed ranges has no impact on the results. The chains typically have worst e-values (the variance(mean)/mean(variance) of $1 / 2$ chains) much smaller than 0.01 , indicating convergence.

In the following, we will discuss the results given by the SNe-only and the $\mathrm{SNe}+\mathrm{CMB}+\mathrm{GC}$ data, respectively.

\section{A. SNe-only cases}

In this subsection, we discuss the results given by the SNe-only data. Notice that the Hubble constant $h$ has been marginalized during the $\chi^{2}$ fitting process of SNe Ia, so we only need to consider six free parameters, including $\alpha, \beta_{0}, \beta_{1}, \Omega_{m}, w_{0}$, and $w_{1}$ [two parameters for the equation of state $w(z)]$. In Table I we list the fitting results for various constant $\beta$ and linear $\beta(z)$ cases, where only SNe data are used. The most obvious feature of this table is that varying $\beta$ can significantly improve the fitting results. This conclusion is insensitive to the dark energy models: for all the models, adding a parameter of $\beta$ can reduce the best-fit values of $\chi^{2}$ by $\sim 36$. Based on the Wilk's theorem, 36 units of $\chi^{2}$ is equivalent to a Gaussian fluctuation of $6 \sigma$. This means that for all the models, the result of $\beta_{1}=0$ is ruled out. It must be stressed that the evolution of $\beta$ is not a special feature only found in the SNLS3 data. In 48, Mohlabeng and Ralston showed that, for the Union2.1 SN data, adding a parameter of $\beta$ can reduce the best-fit values of $\chi^{2}$ by $\sim 50$. Therefore, it is very necessary and important to consider $\beta$ 's evolution in the cosmology-fits.

In addition, we feel that it may be necessary to report the fit results of all the parameters, including the nuisance parameter $\mathcal{M}$, in order for our work to be reproducible for the reader. Therefore, here we give the best-fit values of $\mathcal{M}_{1}$ and $\mathcal{M}_{2}$ for the SNe-only cases. For the constant $\beta$ case: $\mathcal{M}_{1}=0.01327$ and $\mathcal{M}_{2}=$ -0.03708 for the $\Lambda$ CDM model; $\mathcal{M}_{1}=0.01503$ and $\mathcal{M}_{2}=-0.03815$ for the $w \mathrm{CDM}$ model; $\mathcal{M}_{1}=0.01839$ and $\mathcal{M}_{2}=-0.03682$ for the CPL model. For the linear $\beta(z)$ case: $\mathcal{M}_{1}=-0.00343$ and $\mathcal{M}_{2}=-0.05650$ for the $\Lambda \mathrm{CDM}$ model; $\mathcal{M}_{1}=-0.001348$ and $\mathcal{M}_{2}=-0.06815$ for the $w \mathrm{CDM}$ model; $\mathcal{M}_{1}=0.00703$ and $\mathcal{M}_{2}=-0.05333$ for the CPL model.

Let us discuss the SNe-only cases with more details.

\section{- $\Lambda \mathrm{CDM}$ model}

Firstly, we discuss the results of the $\Lambda$ CDM model. In Fig. 1. using SNe-only data, we plot the joint $68 \%$ and $95 \%$ confidence contours for $\left\{\beta_{0}, \beta_{1}\right\}$ (top panel), and the $68 \%, 95 \%$, and $97 \%$ confidence constraints for $\beta(z)$ (bottom panel), for the linear $\beta(z)$ case. For comparison, we also show the best-fit result of constant $\beta$ case on the bottom panel. The top panel shows that $\beta_{1}>0$ at a high 
TABLE I: Fitting results for various constant $\beta$ and linear $\beta(z)$ cases, where only SNe data are used.

\begin{tabular}{|c|c|c|c|c|c|c|}
\hline \multirow[b]{2}{*}{ Parameters } & \multicolumn{2}{|c|}{$\Lambda \mathrm{CDM}$} & \multicolumn{2}{|c|}{$w \mathrm{CDM}$} & \multicolumn{2}{|c|}{$\mathrm{CPL}$} \\
\hline & Const $\beta$ & Linear $\beta(z)$ & Const $\beta$ & Linear $\beta(z)$ & Const $\beta$ & Linear $\beta(z)$ \\
\hline$\alpha$ & $1.425_{-0.103}^{+0.109}$ & $1.410_{-0.094}^{+0.106}$ & $1.427_{-0.101}^{+0.108}$ & $1.410_{-0.092}^{+0.103}$ & $1.427_{-0.106}^{+0.106}$ & $1.415_{-0.097}^{+0.096}$ \\
\hline$\beta_{0}$ & $\begin{array}{r}3.259^{+0.110} \\
-0.108\end{array}$ & $1.457_{-0.376}^{+0.370}$ & $3.256_{-0.102}^{+0.114}$ & $\begin{array}{r}1.439^{+0.398} \\
-0.336\end{array}$ & $3.265_{-0.109}^{+0.104}$ & $1.499_{-0.453}^{+0.300}$ \\
\hline$\beta_{1}$ & $\mathrm{~N} / \mathrm{A}$ & $\begin{array}{c}5.061_{-1.027}^{+1.064} \\
-1.027\end{array}$ & $\mathrm{~N} / \mathrm{A}$ & $5.112_{-1.074}^{+0.970}$ & $\mathrm{~N} / \mathrm{A}$ & $\begin{array}{l}4.939_{-0.796}^{+1.256} \\
-0.79\end{array}$ \\
\hline$\Omega_{m}$ & $0.226_{-0.036}^{+0.040}$ & $0.280_{-0.052}^{+0.052}$ & $0.163_{-0.147}^{+0.100}$ & $0.135_{-0.009}^{+0.215}$ & $0.320_{-0.310}^{+0.055}$ & $0.252_{-0.242}^{+0.137}$ \\
\hline$w_{0}$ & $\mathrm{~N} / \mathrm{A}$ & $\mathrm{N} / \mathrm{A}$ & $-0.858_{-0.224}^{+0.219}$ & $-0.630_{-0.268}^{+0.058}$ & $-0.778_{-0.268}^{+0.235}$ & $-0.667_{-0.240}^{+0.254}$ \\
\hline$w_{1}$ & $\mathrm{~N} / \mathrm{A}$ & $\mathrm{N} / \mathrm{A}$ & $\mathrm{N} / \mathrm{A}$ & $\mathrm{N} / \mathrm{A}$ & $\begin{array}{r}-3.619^{+4.370} \\
-1.380 \\
\end{array}$ & $\begin{array}{r}-2.609 \\
-2.260 \\
-2.739 \\
\end{array}$ \\
\hline$\chi_{m}$ & 420.075 & 385.203 & 419.658 & 383.591 & 419.054 & 383.144 \\
\hline
\end{tabular}

confidence level (CL). Here we adopt $\chi^{2} \equiv \chi_{\min }^{2}+i^{2}$ with $i=1,2$, and 3 , corresponding to $1-3$ units of Gaussian CL $\sigma$. In addition, there is a clear degeneracy between $\beta_{0}$ and $\beta_{1}$, which may be due to the kinematic fact of fitting a linear function. The bottom panel shows that $\beta(z)$ rapidly increases with $z$. Moreover, comparing with the best-fit result of constant $\beta$ case, one can see that $\beta$ deviates from a constant at $6 \sigma \mathrm{CL}$. It needs to be pointed out that the evolutionary behaviors of $\beta(z)$ depends on the SN samples used. In [48, Mohlabeng and Ralston found that, for the Union2.1 SN data, $\beta(z)$ decreases with $z$. This is similar to the case of Pan-STARRS1 SN data 64. It is of great interest to study why different SN data give different evolutionary behaviors of $\beta(z)$, and some numerical simulation studies may be required to solve this problem. We will study this issue in future work.

Now, we study the effects of varying $\beta$ on parameter estimation of $\Lambda \mathrm{CDM}$ model. In Fig. 2, using SNe-only data, we plot the 1D marginalized probability distributions of $\Omega_{m}$ for both the constant $\beta$ and linear $\beta(z)$ cases. We find that varying $\beta$ yields a larger $\Omega_{m}$ : the best-fit result for the constant $\beta$ case is $\Omega_{m}=0.226$, while the best-fit result for the linear $\beta(z)$ case is $\Omega_{m}=0.280$. To make a direct comparison, we also plot the $1 \mathrm{D}$ distribution of $\Omega_{m}$ given by the CMB $+\mathrm{GC}$ data, and find that the best-fit result for this case is $\Omega_{m}=0.287$. Therefore, the result of linear $\beta(z)$ case is much closer to that given by the $\mathrm{CMB}+\mathrm{GC}$ data, compared to the case of treating $\beta$ as a constant. This means that varying $\beta$ is very helpful to reduce the tension between $\mathrm{SNe}$ and other cosmological observations. It should be mentioned that, for different SN data, the effects of varying $\beta$ on parameter estimation are different. For example, for the Union2.1 data, varying $\beta$ yields a smaller $\Omega_{m}$ : the best-fit value of $\Omega_{m}$ is revised from $\Omega_{m}=0.29$ to $\Omega_{m}=0.26$ [4]. For this case, there is no significant tension between SNe and other cosmological observations. This shows that there still exists significant disagreement between different SN samples.

In Fig. 3, using the SNe-only data, we plot the joint $68 \%$ and $95 \%$ confidence contours for $\left\{\Omega_{m}, \beta_{1}\right\}$, for the $\Lambda \mathrm{CDM}$ model. As shown in Table I. $\Omega_{m}$ is related to the value of $\beta_{1}$. To make a direct comparison, we also plot the best-fit point (star symbol) of the constant $\beta$ case, which corresponds to $\chi_{\min }^{2}=420.075$. Compared
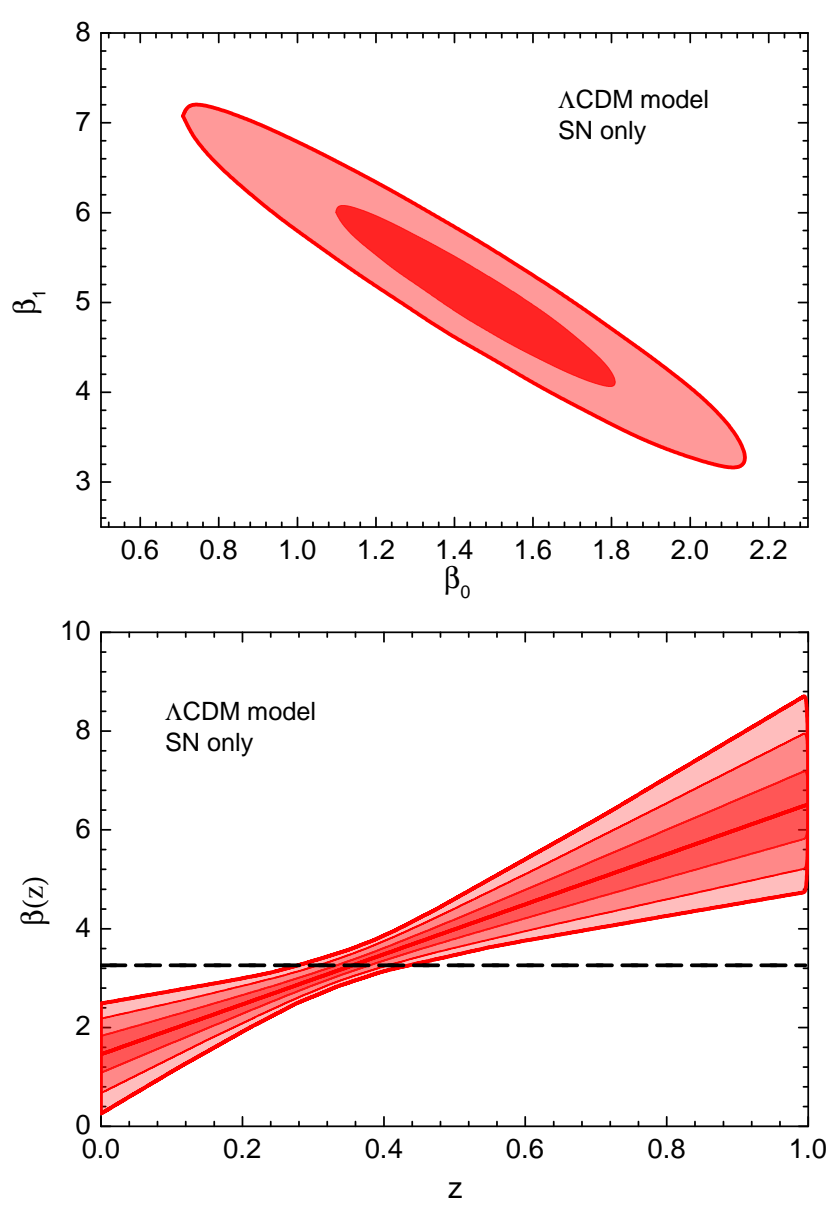

FIG. 1: The joint $68 \%$ and $95 \%$ confidence contours for $\left\{\beta_{0}, \beta_{1}\right\}$ (top panel), and the $68 \%, 95 \%$, and $97 \%$ confidence constraints for $\beta(z)$ (bottom panel), given by the SNe-only data, for the $\Lambda$ CDM model. For comparison, the best-fit result of constant $\beta$ case is also shown on the bottom panel.

with the best-fit point (round point, corresponding to $\left.\chi_{\min }^{2}=385.203\right)$ of the linear $\beta(z)$ case, a constant $\beta$ will enlarge the best-fit value of $\chi^{2}$ by 34.872 , which is equivalent to a Gaussian fluctuation of $5.9 \sigma$. This means that a constant $\beta$ is ruled out at $5.9 \sigma \mathrm{CL}$.

\section{- $w \mathrm{CDM}$ and CPL models}

Next, we discuss the results of the $w$ CDM model and the CPL model. In Fig. 4, using SNe-only data, we plot 


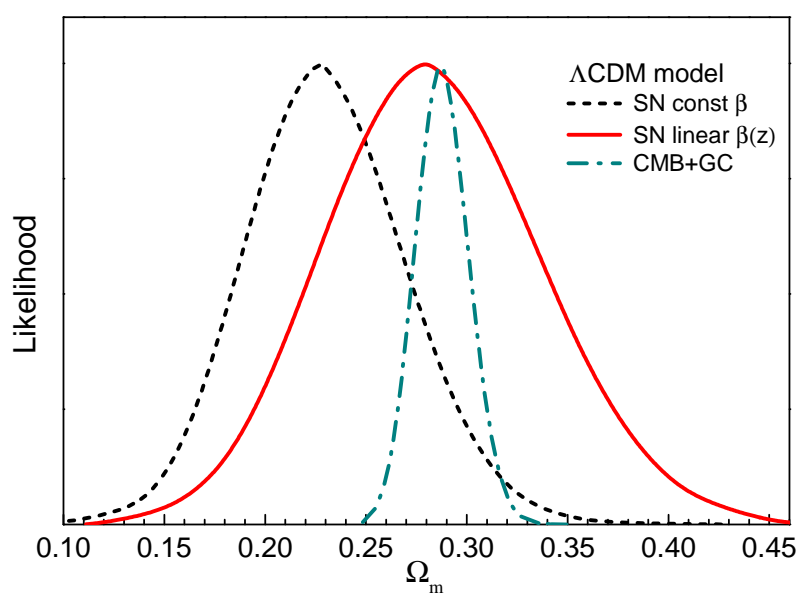

FIG. 2: The 1D marginalized probability distributions of $\Omega_{m}$, given by the SNe-only data, for the $\Lambda \mathrm{CDM}$ model. Both the results of constant $\beta$ and linear $\beta(z)$ cases are presented. The corresponding results given by the $\mathrm{CMB}+\mathrm{GC}$ data are also shown for comparison.

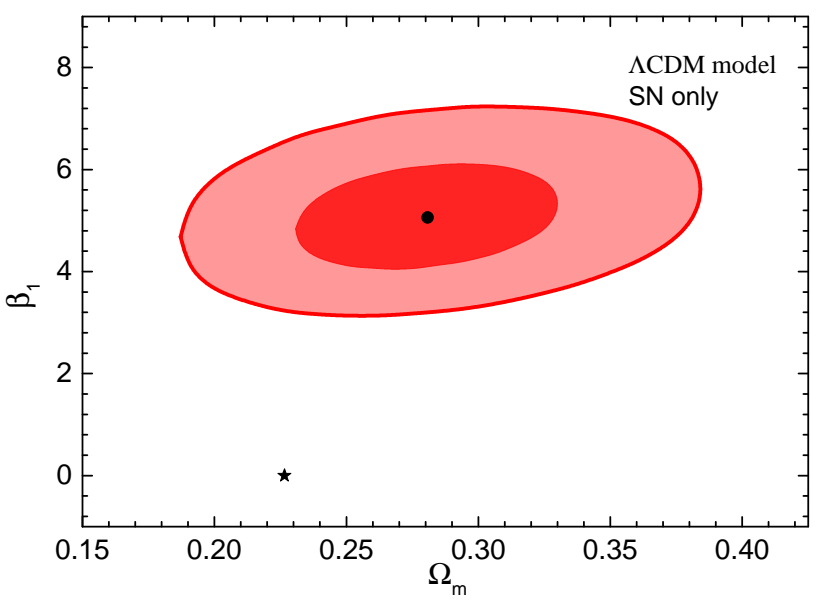

FIG. 3: The joint $68 \%$ and $95 \%$ confidence contours for $\left\{\Omega_{m}, \beta_{1}\right\}$, given by the SNe-only data, for the $\Lambda \mathrm{CDM}$ model. To make a direct comparison, we also plot the best-fit points for the constant $\beta$ (star symbol) and the linear $\beta(z)$ (round point) cases.

the $68 \%, 95 \%$, and $97 \%$ confidence constraints for $\beta(z)$, for the $w$ CDM model (top panel) and the CPL model (bottom panel). It is clear that for both the $w \mathrm{CDM}$ model and the CPL model, $\beta(z)$ rapidly increase with $z$. Moreover, comparing with the best-fit results of constant $\beta$ case, one can see that $\beta$ deviates from a constant at $6 \sigma$ CL. Since the results of Fig. 1 and Fig. 4 are very close, we can conclude that the evolution of $\beta$ is insensitive to the models considered. Based on Table I] one can see that, for both the $w \mathrm{CDM}$ model and the CPL model, varying $\beta$ yields a smaller $\Omega_{m}$, compared to the cases of assuming a constant $\beta$. This result is different from that of the $\Lambda \mathrm{CDM}$ model, and is also different from the results given by the $\mathrm{SNe}+\mathrm{CMB}+\mathrm{GC}$ data (see next subsection). This may be due to using $\mathrm{SNe}$ data alone
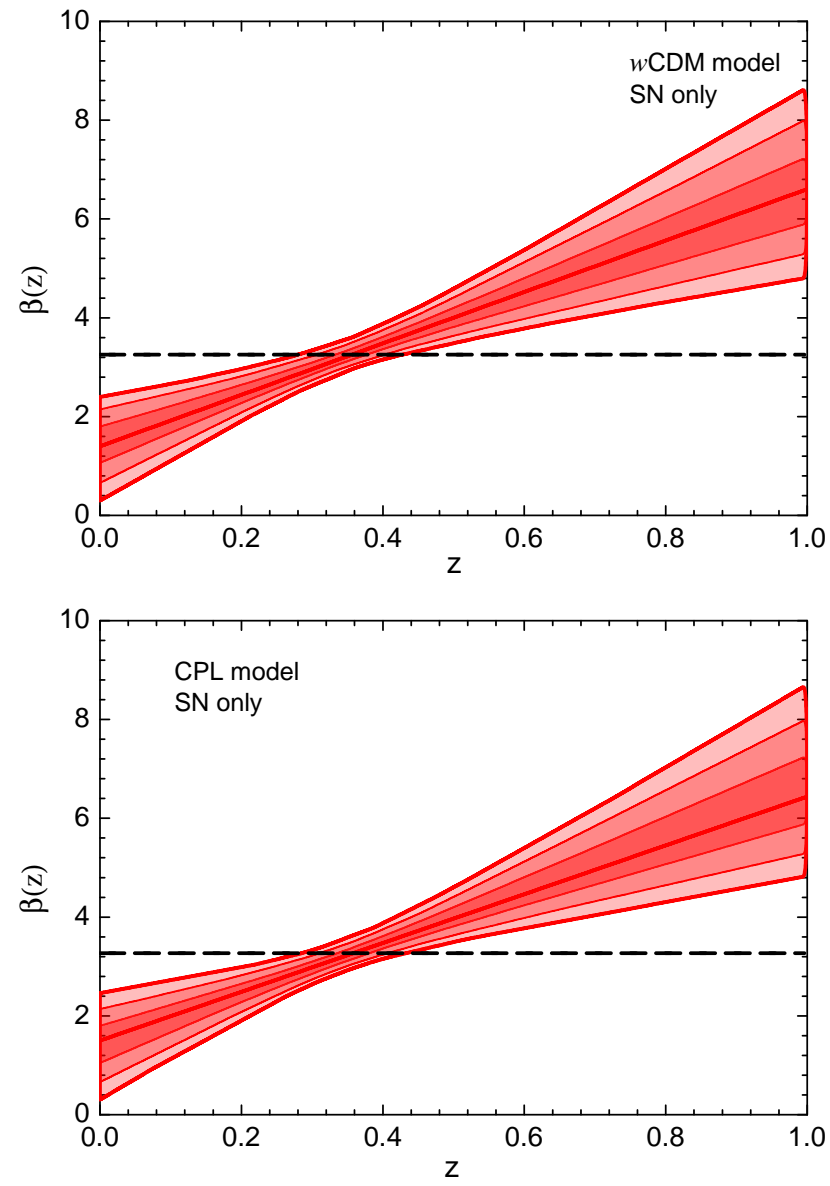

FIG. 4: The $68 \%, 95 \%$, and $97 \%$ confidence constraints for $\beta(z)$, given by the SNe-only data, for the $w \mathrm{CDM}$ model (top panel) and the CPL model (bottom panel). For comparison, the best-fit results of constant $\beta$ cases are also shown.

still has difficulty to break the degeneracy between $\Omega_{m}$ and $w$.

\section{B. $\mathrm{SNe}+\mathrm{CMB}+\mathrm{GC}$ cases}

In this subsection, we discuss the results given by the $\mathrm{SNe}+\mathrm{CMB}+\mathrm{GC}$ data. It should be mentioned that, in order to use the Planck distance priors data, three new model parameters, including $h, \omega_{b}$, and $\Omega_{k}$, must be added. In Table II. we list the fitting results for various constant $\beta$ and linear $\beta(z)$ cases, where the $\mathrm{SNe}+\mathrm{CMB}+\mathrm{GC}$ data are used. Again, we find that varying $\beta$ can significantly improve the fitting results. For all the dark energy models, adding a parameter of $\beta$ can reduce the best-fit values of $\chi^{2}$ by $\sim 36$. This means that after considering the $\mathrm{CMB}$ and the GC data, the result of $\beta_{1}=0$ is still ruled out for all the models. This shows the importance of considering the evolution of $\beta$ in the cosmology-fits.

We also give the best-fit values of $\mathcal{M}_{1}$ and $\mathcal{M}_{2}$ for the $\mathrm{SNe}+\mathrm{CMB}+\mathrm{GC}$ cases. For the constant $\beta$ case: 
TABLE II: Fitting results for various constant $\beta$ and linear $\beta(z)$ cases, where the $\mathrm{SNe}+\mathrm{CMB}+\mathrm{GC}$ data are used.

\begin{tabular}{|c|c|c|c|c|c|c|}
\hline \multirow[b]{2}{*}{ Parameters } & \multicolumn{2}{|c|}{$\Lambda \mathrm{CDM}$} & \multicolumn{2}{|c|}{$w \mathrm{CDM}$} & \multicolumn{2}{|c|}{$\mathrm{CPL}$} \\
\hline & Const $\beta$ & Linear $\beta(z)$ & Const $\beta$ & Linear $\beta(z)$ & Const $\beta$ & Linear $\beta(z)$ \\
\hline$\alpha$ & $\begin{array}{r}1.429_{-0.111}^{+0.099} \\
\end{array}$ & $\begin{array}{c}1.421_{-0.100}^{+0.093} \\
-0.10\end{array}$ & $\begin{array}{r}1.433_{-0.108}^{+0.095} \\
-0.108\end{array}$ & $\begin{array}{r}1.425_{-0.105}^{+0.086} \\
-0.00\end{array}$ & $1.438_{-0.103}^{+0.090}$ & $1.421_{-0.093}^{+0.079}$ \\
\hline$\beta_{0}$ & $3.249_{-0.106}^{+0.109}$ & $1.400_{-0.326}^{+0.394}$ & $\begin{array}{r}3.253_{-0.099}^{+0.109} \\
-0.09\end{array}$ & $1.493_{-0.420}^{+0.300}$ & $3.269_{-0.104}^{+0.099}$ & $1.478_{-0.389}^{+0.258}$ \\
\hline$\beta_{1}$ & $\mathrm{~N} / \mathrm{A}$ & $\begin{array}{r}5.208+0.890 \\
-1.074\end{array}$ & $\mathrm{~N} / \mathrm{A}$ & $\begin{array}{r}4.960_{-0.798}^{+1.089} \\
-0.0\end{array}$ & $\mathrm{~N} / \mathrm{A}$ & $5.012_{-0.735}^{+1.100}$ \\
\hline$\Omega_{m}$ & $0.281_{-0.010}^{+0.013}$ & $0.287_{-0.013}^{+0.011}$ & $0.270_{-0.013}^{+0.014}$ & $0.286_{-0.016}^{+0.013}$ & $0.275_{-0.013}^{+0.012}$ & $0.280_{-0.015}^{+0.013}$ \\
\hline$h$ & $0.704_{-0.014}^{+0.013}$ & $0.698_{-0.012}^{+0.015}$ & $0.719_{-0.018}^{+0.016}$ & $0.698_{-0.016}^{+0.021}$ & $0.714_{-0.015}^{+0.019}$ & $0.706_{-0.016}^{+0.020}$ \\
\hline$\omega_{b}$ & $0.02233_{-0.00030}^{+0.00028}$ & $0.02226_{-0.00026}^{+0.00030}$ & $0.02229_{-0.00028}^{+0.00028}$ & $0.02235_{-0.00034}^{+0.00022}$ & $0.02228_{-0.00028}^{+0.00027}$ & $0.02229_{-0.00028}^{+0.00023}$ \\
\hline$\Omega_{k}$ & $0.0031_{-0.0035}^{+0.0035}$ & $0.0024_{-0.0033}^{+0.0037}$ & $0.0009_{-0.0045}^{+0.0031}$ & $0.0019_{-0.0036}^{+0.0051}$ & $-0.0076_{-0.0033}^{+0.0053}$ & $-0.0093_{-0.0029}^{+0.0054}$ \\
\hline$w_{0}$ & $\mathrm{~N} / \mathrm{A}$ & $\mathrm{N} / \mathrm{A}$ & $-1.091_{-0.085}^{+0.064}$ & $-1.002_{-0.075}^{+0.078}$ & $-0.783_{-0.226}^{+0.162}$ & $-0.619_{-0.209}^{+0.190}$ \\
\hline$w_{1}$ & $\mathrm{~N} / \mathrm{A}$ & $\mathrm{N} / \mathrm{A}$ & $\mathrm{N} / \mathrm{A}$ & $\mathrm{N} / \mathrm{A}$ & $-2.180_{-1.097}^{+1.424}$ & $-3.059_{-1.394}^{+1.610}$ \\
\hline$\chi_{m}^{2}$ & 423.922 & 387.077 & 422.296 & 387.041 & 420.022 & 383.826 \\
\hline
\end{tabular}

$\mathcal{M}_{1}=0.006862$ and $\mathcal{M}_{2}=-0.048252$ for the $\Lambda \mathrm{CDM}$ model; $\mathcal{M}_{1}=0.01078$ and $\mathcal{M}_{2}=-0.03941$ for the $w \mathrm{CDM}$ model; $\mathcal{M}_{1}=0.01878$ and $\mathcal{M}_{2}=-0.03796$ for the CPL model. For the linear $\beta(z)$ case: $\mathcal{M}_{1}=$ -0.003554 and $\mathcal{M}_{2}=-0.05799$ for the $\Lambda$ CDM model; $\mathcal{M}_{1}=-0.003131$ and $\mathcal{M}_{2}=-0.05785$ for the $w \mathrm{CDM}$ model; $\mathcal{M}_{1}=0.008096$ and $\mathcal{M}_{2}=-0.05369$ for the $\mathrm{CPL}$ model.

Let us discuss the effects of varying $\beta$ on various dark energy models in detail.

\section{- $\Lambda$ CDM model}

Firstly, we start from the $\Lambda \mathrm{CDM}$ model. In Fig. 5, using the $\mathrm{SNe}+\mathrm{CMB}+\mathrm{GC}$ data, we plot the $1 \mathrm{D}$ marginalized probability distributions of $\Omega_{m}$ (top panel), and the joint $68 \%$ and $95 \%$ confidence contours for $\left\{\Omega_{m}, h\right\}$ (bottom panel), for the $\Lambda$ CDM model. From the top panel, we see that varying $\beta$ yields a larger $\Omega_{m}$ : the best-fit value of $\Omega_{m}$ for the constant $\beta$ case is 0.281 , while bestfit value of $\Omega_{m}$ for the linear $\beta(z)$ case is 0.287 . To make a direct comparison, we also plot the $1 \mathrm{D}$ distribution of $\Omega_{m}$ given by the $\mathrm{CMB}+\mathrm{GC}$ data. It is clear that the $1 \mathrm{D}$ distribution of $\Omega_{m}$ for the linear $\beta(z)$ case is closer to that given by the $\mathrm{CMB}+\mathrm{GC}$ data. So we can conclude that varying $\beta$ is very helpful to reduce the tension between SNe and other cosmological observations. This conclusion is consistent with that of Fig. 2. From the bottom panel, we see that varying $\beta$ will also yield a smaller $h$ : the best-fit value of $h$ for the constant $\beta$ case is 0.704 , while the best-fit value of $h$ for the linear $\beta(z)$ case is 0.698 . In addition, it is clear that $\Omega_{m}$ and $h$ are anti-correlated.

In Fig. 6, using the $\mathrm{SNe}+\mathrm{CMB}+\mathrm{GC}$ data, we plot the joint $68 \%$ and $95 \%$ confidence contours for $\left\{\Omega_{m}, \beta_{1}\right\}$, for the $\Lambda$ CDM model. As shown in Table II $\Omega_{m}$ is related to the value of $\beta_{1}$. To make a direct comparison, we also plot the best-fit point (star symbol) of the constant $\beta$ case, which corresponds to $\chi_{\min }^{2}=423.922$. Compared with the best-fit point (round point, corresponding to $\left.\chi_{\text {min }}^{2}=387.077\right)$ of the linear $\beta(z)$ case, a constant $\beta$ will enlarge the best-fit values of $\chi^{2}$ by 36.845 , which is equivalent to a Gaussian fluctuation of $6.1 \sigma$. This means that a constant $\beta$ is ruled out at $6.1 \sigma$ CL. Notice that
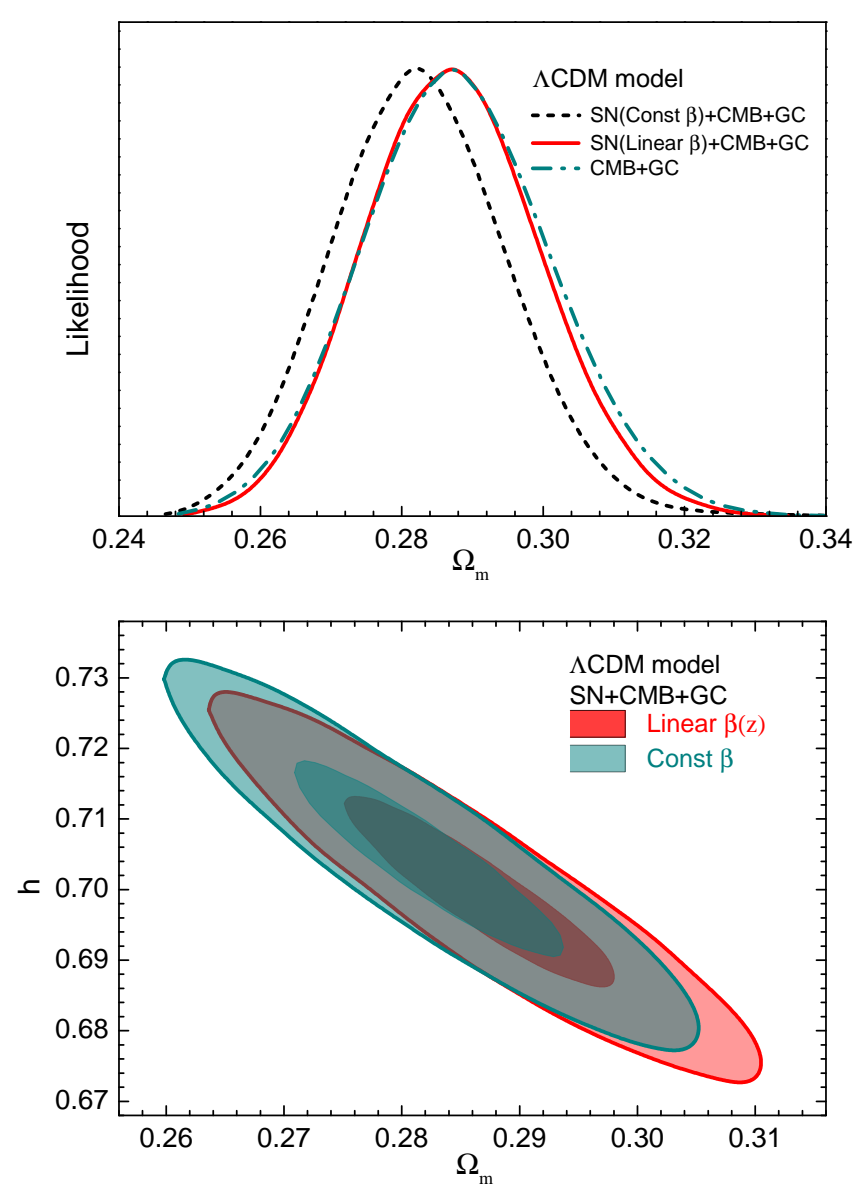

FIG. 5: The 1D marginalized probability distribution of $\Omega_{m}$ (top panel), and the joint $68 \%$ and $95 \%$ confidence contours for $\left\{\Omega_{m}, h\right\}$ (bottom panel), given by the $\mathrm{SNe}+\mathrm{CMB}+\mathrm{GC}$ data, for the $\Lambda \mathrm{CDM}$ model. Both the results of constant $\beta$ and linear $\beta(z)$ cases are shown. The corresponding results given by the $\mathrm{CMB}+\mathrm{GC}$ data are also shown for comparison.

using SNe-only data can only rule out a constant $\beta$ at $5.9 \sigma \mathrm{CL}$, so we can conclude that adding the $\mathrm{CMB}$ and $\mathrm{GC}$ data will strengthen the conclusion of $\beta_{1} \neq 0$.

- $w \mathrm{CDM}$ model 


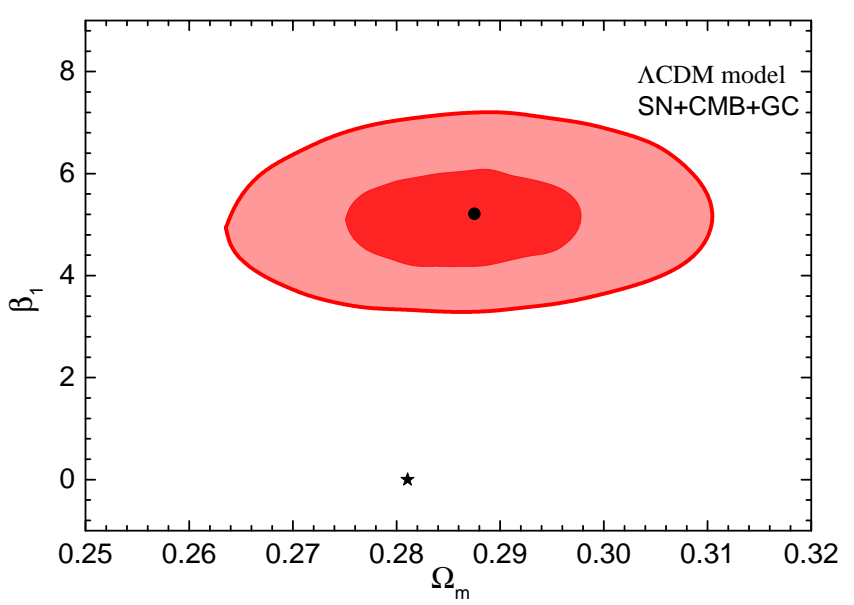

FIG. 6: The joint $68 \%$ and $95 \%$ confidence contours for $\left\{\Omega_{m}, \beta_{1}\right\}$, given by the $\mathrm{SNe}+\mathrm{CMB}+\mathrm{GC}$ data, for the $\Lambda \mathrm{CDM}$ model. To make a direct comparison, we also plot the best-fit points for the constant $\beta$ (star symbol) and the linear $\beta(z)$ (round point) cases.

Then, we turn to the $w \mathrm{CDM}$ model. In Fig. 7, using the $\mathrm{SNe}+\mathrm{CMB}+\mathrm{GC}$ data, we plot the joint $68 \%$ and $95 \%$ confidence contours for $\left\{\Omega_{m}, h\right\}$ (top panel) and $\left\{\Omega_{m}, w_{0}\right\}$ (bottom panel), for the $w \mathrm{CDM}$ model. From the top panel, we see that varying $\beta$ yields a larger $\Omega_{m}$ and a smaller $h$ : the best-fit results for the constant $\beta$ case are $\Omega_{m}=0.270$ and $h=0.719$, while best-fit results for the linear $\beta(z)$ case are $\Omega_{m}=0.286$ and $h=0.698$. In addition, $\Omega_{m}$ and $h$ are anti-correlated. This is consistent with the case of the $\Lambda \mathrm{CDM}$ model. The bottom panel shows that varying $\beta$ will also yield a larger $w_{0}$ : the best-fit value of $w_{0}$ for the constant $\beta$ case is -1.091 , while the best-fit value of $w_{0}$ for the linear $\beta(z)$ case is -1.002 . Notice that after considering the evolution of $\beta$, the results of the $w \mathrm{CDM}$ model are closer to that of the $\Lambda \mathrm{CDM}$ model. In addition, $\Omega_{m}$ and $w_{0}$ are also in positive correlation.

\section{- CPL model}

Next, we discuss the CPL model. In Fig. 8, using the $\mathrm{SNe}+\mathrm{CMB}+\mathrm{GC}$ data, we plot the joint $68 \%$ and $95 \%$ confidence contours for $\left\{\Omega_{m}, h\right\}$ (top panel) and $\left\{\Omega_{m}, w_{0}\right\}$ (bottom panel), for the CPL model. Again, we see from the top panel that varying $\beta$ yields a larger $\Omega_{m}$ and a smaller $h$ : the best-fit results for the constant $\beta$ case are $\Omega_{m}=0.275$ and $h=0.714$, while the bestfit results for the linear $\beta(z)$ case are $\Omega_{m}=0.280$ and $h=0.706$. In addition, $\Omega_{m}$ and $h$ are also anti-correlated. The bottom panel shows that varying $\beta$ will also yield a larger $w_{0}$ : the best-fit value of $w_{0}$ for the constant $\beta$ case is -0.783 , while the best-fit value of $w_{0}$ for the linear $\beta(z)$ case is -0.619 . These results are consistent with the cases of the $\Lambda \mathrm{CDM}$ model and the $w \mathrm{CDM}$ model. To make a direct comparison, we also study the CPL model using the $\mathrm{CMB}+\mathrm{GC}$ data, and find that the bestfit results for this case are $\Omega_{m}=0.282, h=0.709$ and
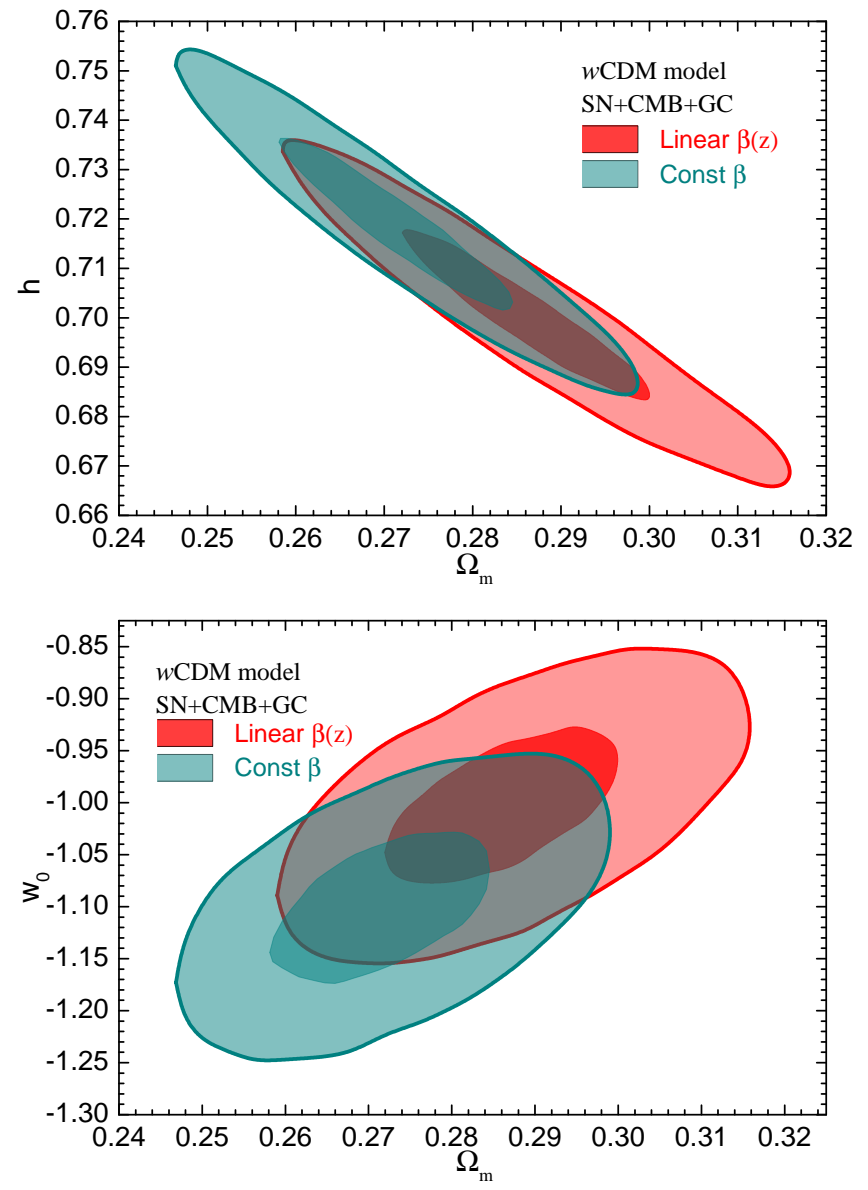

FIG. 7: The joint $68 \%$ and $95 \%$ confidence contours for $\left\{\Omega_{m}, h\right\}$ (top panel) and $\left\{\Omega_{m}, w_{0}\right\}$ (bottom panel), given by the $\mathrm{SNe}+\mathrm{CMB}+\mathrm{GC}$ data, for the $w \mathrm{CDM}$ model. Both the results of constant $\beta$ and linear $\beta(z)$ cases are shown for comparison.

$w_{0}=-0.712$. It is clear that the fitting results for the linear $\beta(z)$ case are much closer to that given by the $\mathrm{CMB}+\mathrm{GC}$ data, compared to the case of treating $\beta$ as a constant. This indicates that the conclusion of Figs. 2 and 5 is insensitive to the dark energy models considered.

Finally, we discuss the effects of varying $\beta$ on the equation of state (EOS) $w(z)$ of the CPL model. In Fig. 9 , using the $\mathrm{SNe}+\mathrm{CMB}+\mathrm{GC}$ data, we plot the joint $68 \%$ and $95 \%$ confidence contours for $\left\{w_{0}, w_{1}\right\}$ (top panel), and the $68 \%$ and $95 \%$ confidence constraints for $w(z)$ (bottom panel), for the CPL model. The top panel shows that varying $\beta$ yields a larger $w_{0}$ and a smaller $w_{1}$, while $w_{0}$ and $w_{1}$ are anti-correlated. The bottom panel shows that after considering the evolution of $\beta, \operatorname{EOS} w(z)$ of the CPL model will decrease faster with redshift $z$.

\section{DISCUSSION AND SUMMARY}

Along with the rapid progress of SN cosmology, more and more SNe Ia have been discovered, and the systematic errors of SNe Ia have drawn more and more 

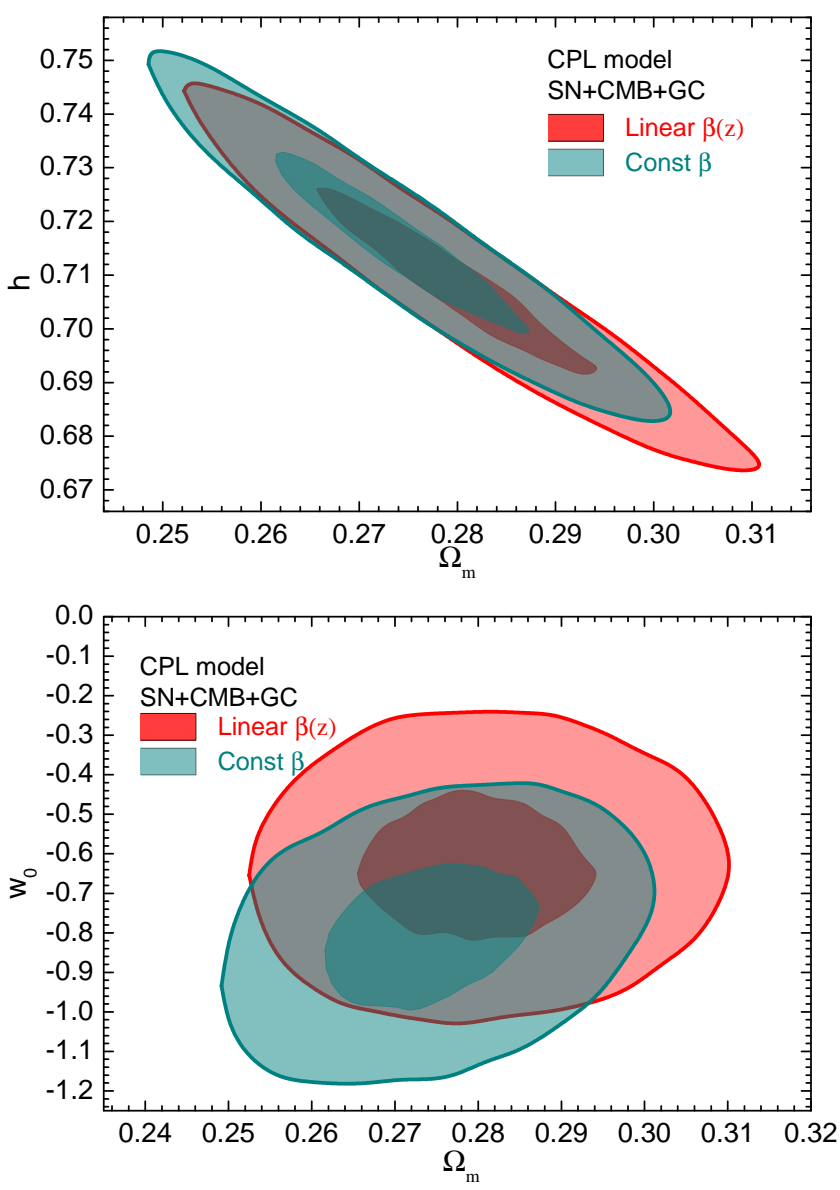

FIG. 8: The joint $68 \%$ and $95 \%$ confidence contours for $\left\{\Omega_{m}, h\right\}$ (top panel) and $\left\{\Omega_{m}, w_{0}\right\}$ (bottom panel), given by the $\mathrm{SNe}+\mathrm{CMB}+\mathrm{GC}$ data, for the CPL model. Both the results of constant $\beta$ and linear $\beta(z)$ cases are shown for comparison.

attentions. One of the most important systematic uncertainties for SNe Ia is the potential SN evolution. The hints for the evolution of $\beta$ have been found in [38, 39, 41, 44, 46, 47, but these papers explored $\beta$ 's evolution using bin-by-bin fits, which were very difficult to make definitive statements because of the correlations between different bins. In [48, Mohlabeng and Ralston firstly used a linear parametrization $\beta(z)=\beta_{0}+\beta_{1} z$ to study the Union2.1 sample, and found that $\beta$ deviates from a constant at $7 \sigma$ confidence levels. Moreover, they proved that using a linear parametrization form can obtain better results than using bin-by-bin methods. Wang and Wang [4] studied the case of SNLS3 data using three functional forms, and also found strong evidence for the redshift-evolution of $\beta$.

It is clear that a time-varying $\beta$ will have significant impact on parameter estimation. So in this paper, by adopting a constant $\alpha$ and a linear $\beta(z)=\beta_{0}+\beta_{1} z$, we have further explored the evolution of $\beta$ and its effects on parameter estimation. To perform the cosmology-fits, we have considered three simplest dark energy models: $\Lambda \mathrm{CDM}, w \mathrm{CDM}$, and CPL. In addition to the SNLS3 SN
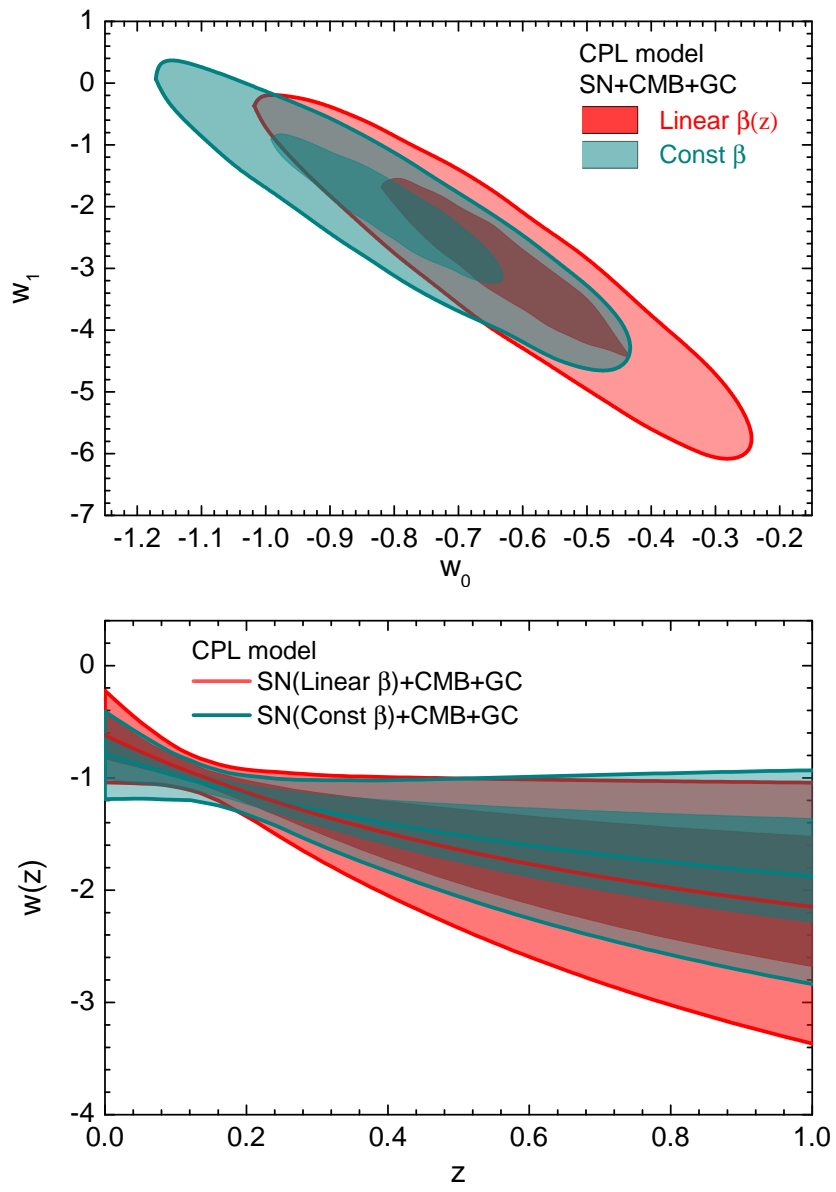

FIG. 9: The joint $68 \%$ and $95 \%$ confidence contours for $\left\{w_{0}, w_{1}\right\}$ (top panel), and the $68 \%$ and $95 \%$ confidence constraints for $w(z)$ (bottom panel), given by the $\mathrm{SNe}+\mathrm{CMB}+\mathrm{GC}$ data, for the $\mathrm{CPL}$ model. Both the results of constant $\beta$ and linear $\beta(z)$ cases are shown for comparison.

data, we have also taken into account the Planck distance priors data, as well as the latest GC data extracted from SDSS DR7 and BOSS.

We further confirm the redshift-evolution of $\beta$ for the SNLS3 data: For all the models, adding a parameter of $\beta$ can reduce $\chi_{\text {min }}^{2}$ by $\sim 36$, indicating that $\beta_{1}=0$ is ruled out at $6 \sigma$ CL. In other words, $\beta$ deviates from a constant at $6 \sigma \mathrm{CL}$. This conclusion is insensitive to the dark energy models considered and the SN data used, showing the importance of considering the evolution of $\beta$ in the cosmology-fits.

Furthermore, it is found that varying $\beta$ can significantly change the fitting results of various cosmological parameters: using the SNLS3 data alone, varying $\beta$ yields a larger $\Omega_{m}$ for the $\Lambda$ CDM model; using the SNLS3 + CMB + GC data, varying $\beta$ yields a larger $\Omega_{m}$ and a smaller $h$ for all the models. For the $w \mathrm{CDM}$ model, varying $\beta$ will also yield a larger $w_{0}$; for the CPL model, varying $\beta$ yields a larger $w_{0}$ and a smaller $w_{1}$. Moreover, we find that these results are much closer to those given by the $\mathrm{CMB}+\mathrm{GC}$ data, compared to the cases of 
treating $\beta$ as a constant. This indicates that considering the evolution of $\beta$ is very helpful for reducing the tension between supernova and other cosmological observations.

In this paper, only three simplest dark energy models are considered. It is of interest to study the effects of varying $\beta$ on parameter estimation in other dark energy models. In addition, some other factors, such as the evolution of $\sigma_{\text {int }}$ 54, may also cause the systematic uncertainties of SNe Ia. These issues will be studied in future works.

\section{Acknowledgments}

We thank the referee for valuable suggestions, which help us to improve this work significantly. We also thank
Dr. Daniel Scolnic for helpful discussions. We are grateful to Dr. Alex Conley for providing us with the SNLS3 covariance matrices that allow redshift-dependent $\beta$. We acknowledge the use of CosmoMC. This work is supported by the National Natural Science Foundation of China (Grants No. 10975032 and No. 11175042) and by the National Ministry of Education of China (Grants No. NCET-09-0276 and No. N120505003).
[1] A. G. Riess et al., AJ. 116, 1009 (1998); S. Perlmutter et al., ApJ. 517, 565 (1999).

[2] D. N. Spergel et al., ApJS 148, 175 (2003); C. L. Bennet et al., ApJS. 148, 1 (2003); D. N. Spergel et al., ApJS 170, 377 (2007); L. Page et al., ApJS 170, 335 (2007); G. Hinshaw et al., ApJS 170, 263 (2007).

[3] M. Tegmark et al., Phys. Rev. D 69, 103501 (2004); ApJ 606, 702 (2004); Phys. Rev. D 74, 123507 (2006).

[4] E. Komatsu et al., ApJS. 180, 330 (2009); E. Komatsu et al., ApJS. 192, 18 (2011).

[5] W. J. Percival et al., MNRAS 401, 2148 (2010); A. G. Sanchez, et al., arXiv:1203.6616, MNRAS accepted.

[6] M. Drinkwater et al., MNRAS 401, 1429 (2010); C. Blake et al., arXiv:1108.2635, MNRAS accepted.

[7] A. G. Riess et al., ApJ. 730, 119 (2011).

[8] P. J. E. Peebles and B. Ratra, ApJ 325, L17 (1988); C. Wetterich, Nucl. Phys. B 302, 668 (1988); R. R. Caldwell, R. Dave and P. J. Steinhardt, Phys. Rev. Lett. 80, 1582 (1998); I. Zlatev, L. Wang and P. J. Steinhardt, Phys. Rev. Lett. 82, 896 (1999).

[9] R. R. Caldwell, Phys. Lett. B 545, 23 (2002); S. M. Carroll, M. Hoffman and M. Trodden, Phys. Rev. D 68, 023509 (2003); R. R. Caldwell, M. Kamionkowski and N. N. Weinberg, Phys. Rev. Lett. 91, 071301 (2003).

[10] C. Armendariz-Picon, T. Damour and V. Mukhanov, Phys. Lett. B 458, 209 (1999); C. Armendariz-Picon, V. Mukhanov and P. J. Steinhardt, Phys. Rev. D 63, 103510 (2001); T. Chiba, T. Okabe and M. Yamaguchi, Phys. Rev. D 62, 023511 (2000).

[11] A. Y. Kamenshchik, U. Moschella and V. Pasquier, Phys. Lett. B 511, 265 (2001); M. C. Bento, O. Bertolami and A. A. Sen, Phys. Rev. D 66, 043507 (2002).

[12] T. Padmanabhan, Phys. Rev. D 66, 021301 (2002); J. S. Bagla, H. K. Jassal, and T. Padmanabhan, Phys. Rev. D 67, 063504 (2003).

[13] M. Li, Phys. Lett. B 603, 1 (2004); Q. G. Huang and M. Li, JCAP 08, 013 (2004). X. Zhang and F. Q. Wu, Phys. Rev. D 72, 043524 (2005); Phys. Rev. D 76, 023502 (2007); M. Li, C. S. Lin and Y. Wang, JCAP 05, 023 (2008); M. Li, X. D. Li, S. Wang and X. Zhang, JCAP 06, 036 (2009); M. Li et al., JCAP 12, 014 (2009); Y. H. Li, S. Wang, X. D. Li and X. Zhang, JCAP 02, 033
(2013); M. Li, X. D. Li, Y. Z. Ma, X. Zhang and Z. H. Zhang, JCAP 09, 021 (2013).

[14] H. Wei, R. G. Cai, and D. F. Zeng, Class. Quant. Grav. 22, 3189 (2005); H. Wei, and R. G. Cai, Phys. Rev. D 72, 123507 (2005); H. Wei, N. Tang, and S. N. Zhang, Phys. Rev. D75, 043009 (2007).

[15] W. Zhao and Y. Zhang, Class. Quant. Grav. 23, 3405 (2006); T. Y. Xia and Y. Zhang, Phys. Lett. B 656, 19 (2007); S. Wang, Y. Zhang and T. Y. Xia, JCAP 10, 037 (2008); S. Wang and Y. Zhang, Phys. Lett. B 669, 201 (2008).

[16] K. Freese et al., Nucl.Phys. B 287, 797 (1987); A. Linde, in Three hundred years of gravitation, (Eds.: Hawking, S.W. and Israel, W., Cambridge Univ. Press, 1987), 604; J. A. Frieman, C. T. Hill, A. Stebbins, and I. Waga, Phys. Rev. Lett. 75, 2077 (1995); M. Chevallier and D. Polarski, Int. J. Mod. Phys. D 10, 213 (2001); E. V. Linder, Phys. Rev. Lett. 90091301 (2003); D. Huterer and G. Starkman, Phys. Rev. Lett. 90, 031301 (2003); D. Huterer and A. Cooray, Phys. Rev. D 71, 023506 (2005); A. Shafieloo, V. Sahni and A. A. Starobinsky, Phys. Rev. D 80, 101301(R) (2009).

[17] Y. Wang and M. Tegmark, Phys. Rev. Lett. 92, 241302 (2004); Y. Wang, and K. Freese, Phys. Lett. B 632, 449 (2006); Y. Wang and P. Mukherjee, ApJ. 650, 1 (2006); Y. Wang and P. Mukherjee, Phys. Rev. D 76, 103533 (2007); Y. Wang,Phys. Rev. D 78, 123532 (2008).

[18] Y. Wang and M. Tegmark, Phys. Rev. D 71, 103513 (2005);

[19] Q. G. Huang, M. Li, X. D. Li and S. Wang, Phys. Rev. D 80, 083515 (2009); S. Wang, X. D. Li and M. Li, Phys. Rev. D 82, 103006 (2010); M. Li, X. D. Li and X. Zhang, Sci. China Phys. Mech. Astron. 53, 1631 (2010); S. Wang, X. D. Li and M. Li, Phys. Rev. D 83, 023010 (2011); X. D. Li et al., JCAP 07, (2011) 011; J. Z. Ma and X. Zhang, Phys. Lett. B 699, 233 (2011); H. Li and X. Zhang, Phys. Lett. B 713, 160 (2012); X. D. Li, S. Wang, Q. G. Huang, X. Zhang and M. Li, Sci. China Phys. Mech. Astron. 55, 1330 (2012).

[20] V. Sahni and S. Habib, Phys. Rev. Lett. 81, 1766 (1998).

[21] L. Parker and A. Raval, Phys. Rev. D 60, 063512 (1999).

[22] G. Dvali, G. Gabadadze and M. Porrati, Phys. Lett. B 
485, 208 (2000).

[23] S. Nojiri, S. D. Odintsov, and M. Sasaki, Phys. Rev. D 71, 123509 (2005).

[24] A. Nicolis, R. Rattazzi, and E. Trincherini, Phys. Rev. D 79, 064036 (2009).

[25] W. Hu and I. Sawicki, Phys. Rev. D 76, 064004 (2007); A. A. Starobinsky, J. Exp. Theor. Phys. Lett. 86, (2007) 157.

[26] G. R. Bengochea and R. Ferraro, Phys. Rev. D 79, 124019 (2009); E. V. Linder, Phys. Rev. D 81, (2010) 127301.

[27] T. Harko, F. S. N. Lobo, S. Nojiri and S. D. Odintsov, Phys. Rev. D 84, 024020 (2011).

[28] E. J. Copeland, M. Sami and S. Tsujikawa, Int. J. Mod. Phys. D 15, 1753 (2006).

[29] J. Frieman, M. Turner and D. Huterer, Ann. Rev. Astron. Astrophys 46, 385 (2008).

[30] E. V. Linder, Rept. Prog. Phys. 71, 056901 (2008).

[31] R. R. Caldwell and M. Kamionkowski, Ann. Rev. Nucl. Part. Sci. 59, 397 (2009).

[32] J.-P. Uzan, arXiv:0908.2243

[33] S. Tsujikawa, arXiv:1004.1493

[34] S. Nojiri and S. D. Odintsov, Phys. Rept. 505, 59 (2011).

[35] M. Li, X. D. Li, S. Wang and Y. Wang, Commun. Theor. Phys. 56, 525 (2011).

[36] T. Clifton, P. G. Ferreira, A. Padilla and C. Skordis, Phys. Rept. 513, 1 (2012).

[37] Y. Wang, Dark Energy, Wiley-VCH (2010).

[38] Astier, et al., Astron. Astrophys. 447, 31 (2006).

[39] M. Kowalski, et al., ApJ. 686, 749 (2008).

[40] M. Hicken, et al., ApJ. 700, 1097 (2009); M. Hicken, et al., ApJ. 700, 331 (2009).

[41] R. Kessler, et al., ApJS. 185, 32 (2009).

[42] R. Amanullah, et al., ApJ. 716, 712 (2010).

[43] N. Suzuki, et al., ApJ 746, 85 (2012).

[44] J. Guy, et al., A\&A, 523, 7 (2010).

[45] A. Conley, et al., ApJS. 1921 (2011) - C11

[46] M. Sullivan, et al., arXiv:1104.1444.

[47] Marriner, et al., arXiv:1107.4631

[48] G.Mohlabeng and J. Ralston, arXiv:1303.0580

[49] S. Wang and Y. Wang, Phys. Rev. D 88, 043511 (2013).

[50] M. Chevallier and D. Polarski, Int. J. Mod. Phys. D 10, 213 (2001); E. V. Linder, Phys. Rev. Lett. 90, 091301 (2003).

[51] Y. Wang and S. Wang, Phys. Rev. D 88, 043522 (2013).

[52] C. H. Chuang and Y. Wang, MNRAS, 426, 226 (2012).

[53] C. H. Chuang, et al., arXiv:1303.4486

[54] A. Kim, arXiv:1101.3513; J. Marriner, et al., arXiv:1107.4631

[55] Y. Wang, ApJ 536, 531 (2000).

[56] Y. Wang and P. Mukherjee, ApJ. 606, 654 (2004).

[57] Y. Wang, JCAP, 03, 005 (2005).

[58] Y. Wang, C. H. Chuang and P. Mukherjee, Phys. Rev. D 85, 023517 (2012).

[59] Y. Wang and P. Mukherjee, Phys. Rev. D, 76, 103533 (2007).

[60] Y. Wang, Phys. Rev. D 77, 123525 (2008).

[61] W. Hu and N. Sugiyama, ApJ, 471, 542 (1996).

[62] D. Eisenstein and W. Hu, ApJ, 496, 605 (1998).

[63] A. Lewis and S. Bridle, Phys. Rev. D 66, 103511 (2002).

[64] D. Scolnic, et al., arXiv:1310.3824 